\title{
Characterization of the patellar tendon reflex response using an indigenously developed system and implementation of a strategic protocol to assess its clinical usefulness
}

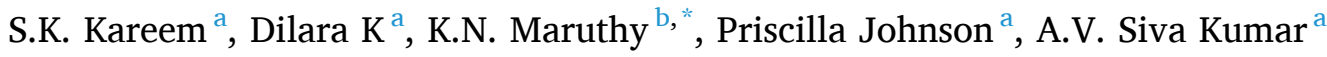 \\ ${ }^{a}$ Department of Physiology, Sri Ramachandra Institute of Higher Education and Research, Porur, Chennai, India \\ ${ }^{\mathrm{b}}$ Department of Physiology, Narayana Medical College and Hospital, Nellore, India
}

\section{A R T I C L E I N F O}

\section{Keywords:}

Muscle stretch reflex

Wearable biosensors

Impact force

Knee excursion

Angular displacement

\begin{abstract}
A B S T R A C T
Introduction: Deep tendon reflex evaluation is a part of the neurological examination and a wide variety of neurological and neuromuscular disorders manifest alterations in deep tendon reflex responses. Conventional methods to assess tendon reflexes in clinical practice are subjective and provide categorical data that might not be adequate in the interpretation. Hence, a quantitative tool is warranted to provide an objective outcome of tendon reflex assessment. The goal of this work was to use an indigenously designed method to characterize the tendon reflex responses.

Materials \& methods: A cross-sectional study was undertaken in 40 healthy subjects aged between 20 and $45 y e a r s$. Assessment of the tendon reflex was performed using wearable biosensors to characterize it into quantitative variables. A strategic protocol was followed to test the accuracy and repeatability of the outcome.

Results: Analysis of phase - I results of the subjective assessment showed high interrater variability and significant bilateral PTR test variability. Whereas the results of the objective assessment showed that the bilateral PTR test variability is minimal by both the assessors. However, the results of all quantified PTR test variables showed an insignificant inter-observer variability except for T 1 , TTE and PEAD ( $\mathrm{p}<0.05^{*}$ ).

Conclusion: The findings of this study suggest that quantified tendon reflex using wearable biosensors could provide a piece of valuable information during neurological examination in clinical settings. The proposed tendon reflex quantification tool is non - invasive, portable and inexpensive that can be used for field studies in different clinical conditions.
\end{abstract}

\section{Introduction}

Reflex is a inborn, involuntary and stereotypic response to a stimulus, and for any reflex to be present, an intact reflex arc is a must. ${ }^{1}$ The evaluation of deep tendon reflexes (DTR) is one of the essential components in clinical examination of the nervous system. Assessment of these tendon reflexes is an essential clinical tool for diagnosing and localizing neurological and neuromuscular disorders. ${ }^{2},{ }^{3}$ Patellar tendon reflex (PTR) is a simple and conveniently performed tendon reflex test. ${ }^{4}$ Pathological alterations in these reflexes are important signs of neurological diseases and distinguishing between delayed or exaggerated PTR responses may help to detect neurological disorders. ${ }^{5},{ }^{6}$ The reflex latency and amplitude of PTR response may vary in people with neurological, neuromuscular, and endocrine disorders. ${ }^{7},{ }^{8}$ Therefore, observation of irregularities in kinematics of tendon refelexes provides valuable insight into the diagnosis and prognosis of the patient's neurological condition. However, the conventional approach to assess the tendon reflexes using semi-quantitative reflex grading scales depends on the examiner's visual judgment. ${ }^{9},{ }^{10}$ NINDS (National Institute of Neurological Disorders and Stroke) and Mayo clinic reflex grading scales are two widely accepted ordinal scales utilized for the PTR assessment. ${ }^{11}$ But, these scales lack temporal data and have high inter-rater variability. ${ }^{12},{ }^{13}$ Therefore, improved tools and techniques are required to quantify PTR responses accurately to enhance the utility of DTR examination. The objective of this study was to improve the clinical utility of DTR examination by quantifying the PTR kinematic features

\footnotetext{
* Corresponding author.

E-mail addresses: kareem.sb9@gmail.com (S.K. Kareem), dilarak@sriramachandra.edu.in (D. K), dr.maruthy@gmail.com (K.N. Maruthy), priscillajohnson@ sriramachandra.edu.in (P. Johnson), reddy.sivakumar5@gmail.com (A.V.S. Kumar).
} 
using limb - mounted biomechanical sensors in normal healthy volunteers.

\section{Methods}

In this descriptive cross-sectional study, a sample of convenience of 40 healthy volunteers were recruited ( 25 males and 15 females, age range $20-45$ years). The participants of the study were recruited from the staff, students and the individuals who attended the outpatient department of Narayana medical college, Nellore for a master health checkup. All the participants were free from disease or disability of the lower limb with no history of neurological, musculoskeletal, or endocrine disorders. Written informed consent was obtained from all participants in accordance with the Declaration of Helsinki, and the study protocol was approved by the Institution Ethics Committee at Sri Ramchandra Institute of Higher Education and Research, Chennai (Reference No: IEC - NI/18/SEP/66/49). The study information leaflet was designed to explain the study's protocol and the experimental procedure was described verbally to the participants.

\subsection{PTR response quantification system}

The proposed in-house built system is constructed based on wearable biosensors that gather biomechanical movement data. Our previous work describes the design and development strategy of the PTR quantification system consisting of an uni-axial accelerometer, electrogoniometer and impact hammer with a load cell. ${ }^{14}$ A similar kind of handheld knee hammer was used in a previous study using a force sensor to quantify tendon reflexes in normal and spastic limbs. ${ }^{15}$ Electrogoniometer is an electronic version of the standard goniometer used to measure joint range of motion (RoM) or angular displacement. Electrogoniometer used in this study consists of two freely movable arms and a potentiometer (3590S Precision Multi-turn Potentiometer). Whenever the angle between the two arms of the electrogoniometer changes, the potentiometer's resistance also changes. This change in resistance is proportional to angular displacement at the knee and measured in the form of the voltage output by the data acquisition system (DAQ). The voltage signals obtained from the electrogoniometer were converted and expressed as degrees. The uniaxial accelerometer is based on the ADXL335 model by Analog devices with signal conditioned voltage output. The analog voltage signals from the biosensors were obtained and processed by Analog to Digital Converter (ADC) module into digital data. All the output signals from the PTR quantification system were converted to digital signals and were captured in real-time by a PC-based data acquisition application (SmartDAQ, V 1.3 by iCircuit Technologies) which is a free and open-source application. Output signals from biosensors were sampled at a rate of $1000 \mathrm{~Hz}$ with $1-\mathrm{ms}$ accuracy. The data acquisition system (Fig. 1) stores the data of the PTR test in a text file format. The digital data was then exported to Microsoft Excel for further analysis of PTR response and output signals from the biosensors were plotted as a waveform (Fig. 2).

\subsection{Experimental procedure}

To assess the precision and accuracy of the PTR quantification system, intra-observer and inter-observer variability was assessed. In this study, the results of the NINDS scale were compared with the quantified PTR results. The assessment of the PTR test was carried out under two phases by two groups of examiners. In the first phase of evaluation, the PTR test was evaluated by two experienced clinicians using the NINDS reflex rating scale and the second phase encompassed the objective assessment of the PTR test by two trained assessors using the PTR quantification system.

\subsubsection{Phase - I assessment}

During this phase of assessment, each participant was evaluated for PTR response by clinicians using the ordinal NINDS reflex rating scale and the PTR responses were graded according to the scale. The clinicians used a traditional Taylor hammer to strike the patellar tendon to elicit the reflex response. The tendon striking force was difficult to quantify using a traditional hammer. Therefore, to maintain an approximately constant striking force on the tendon, pre-test training was given to the clinicians. During the assessment of the PTR response magnitude, the clinicians were recommended to focus on the change of the knee angle as suggested by Dafkin et al. (2012) Clinicians were requested to grade their observations of PTR response magnitude using the conventional five-point reflex grading scale categorized into grades (0-4). Grade 0: Reflex absent; Grade 1: Slight response; Grade 2: Reflex in the lower half of normal; Grade 3: Reflex in the upper half of normal; Grade 4: Enhanced reflex, i.e., clonus. ${ }^{16},{ }^{17}$ Three trials were done on each participant from which the best response was graded by clinicians according to their visual judgement.

\subsubsection{Phase - II assessment}

In this phase, bilateral PTR responses were evaluated using PTR quantification system by two independent trained assessors. The

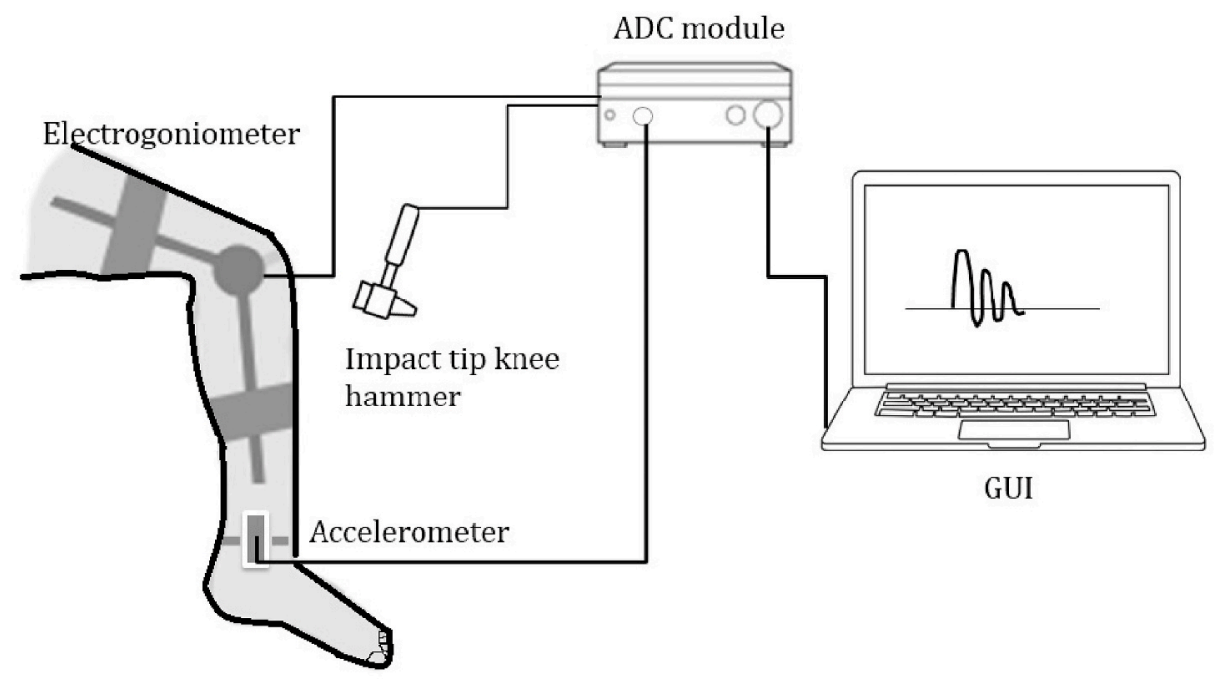

Fig. 1. Experimental setup of the PTR quantification system

Fig. 1: Schematic representation of the setup of the PTR quantification system with Graphical user interface (GUI). 


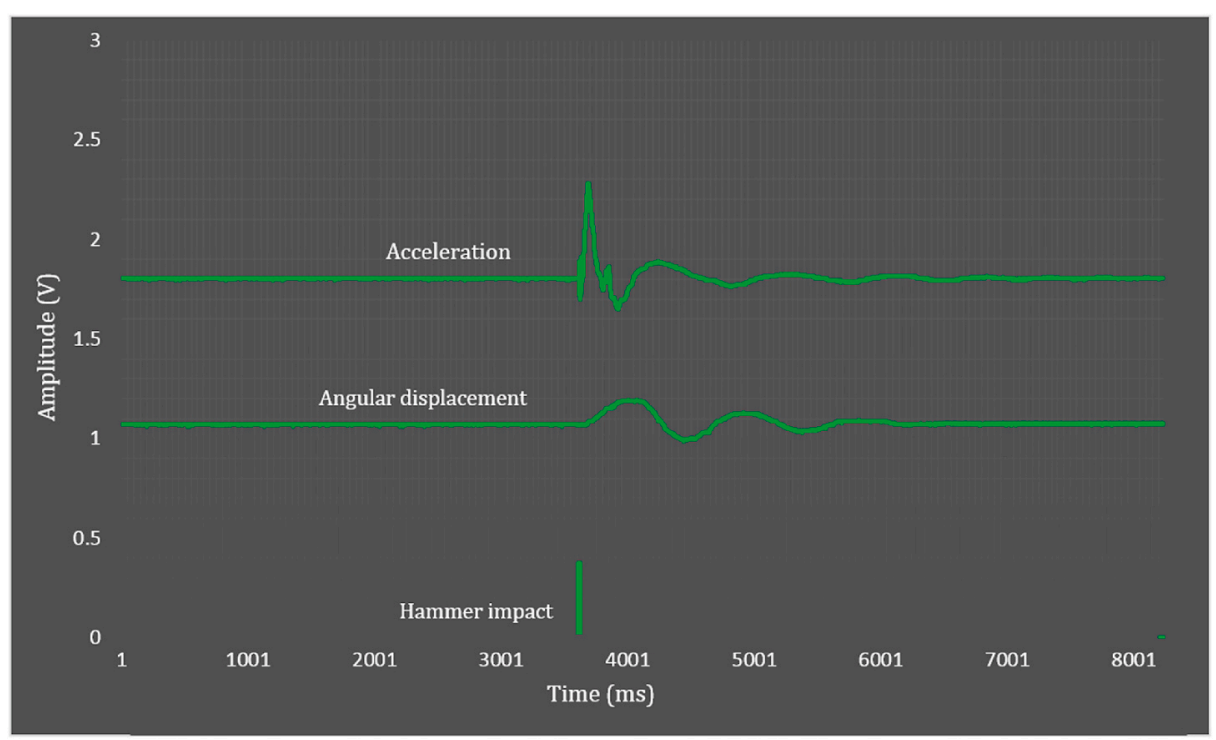

Fig. 2. Graphical recording of PTR response

Fig. 2: Quantified PTR response comprising the data from Accelerometer (Acceleration of limb), Electrogoniometer (Angular displacement) and Hammer impact as a waveform. The output from biosensors was recorded as amplitude in volts with respect to time.

wearable biosensors of the PTR quantification system were fixed at a specified position on the leg using an elastic strap, as illustrated in Fig. 1. Each participant was made to sit comfortably on an adjustable chair, which allows their legs to swing freely. PTR response was elicited by a quick and sharp strike delivered over the patellar tendon with a customized handheld knee hammer. The assessors were recommended to maintain tapping angles approximately at $45^{\circ}$ to $90^{\circ}$ to increase the uniformity in striking the patellar tendon as concluded by the previous study. ${ }^{18}$ On each participant, five PTR test trails (On each knee) were done, from which the response comprising of the largest amplitude with maximum impact force was selected for further analysis. Between each PTR measurement, an interval of 5 min was implemented to avoid fatigue and habituation. During both phases, the PTR assessment was carried out without applying the reinforcement technique (Jendrassik's manoeuvre). During phase - II PTR assessment, surface electromyography (SEMG) of quadriceps femoris was also obtained simultaneously to measure reflex latency and reflex mediated EMG peak amplitude of PTR response. The EMG response of the quadriceps was obtained using bipolar SEMG sensors. The SEMG sensors were fixed on the belly of the quadriceps following skin preparation to enhance electrode-skin contact according to the recommendations of SENIAM (Surface electromyography for the non-invasive assessment of muscles). ${ }^{19}$

\subsection{Data treatment and characteristics of PTR response}

The dynamic parameters of PTR such as Movement latency (ML), Total PTR response time (TPTR), Peak extension angular displacement (PEAD), Peak flexion angular displacement (PFAD), Angular velocity (AV), Time delay to first peak $\left(\Delta \mathrm{t}_{1}\right)$, Reflex mediated EMG peak amplitude and Tendon tapping force (TTF) were measured. Kinematic response of the knee during the test was measured as knee angular displacement with respect to time (Fig. 3) to derive angular velocity.

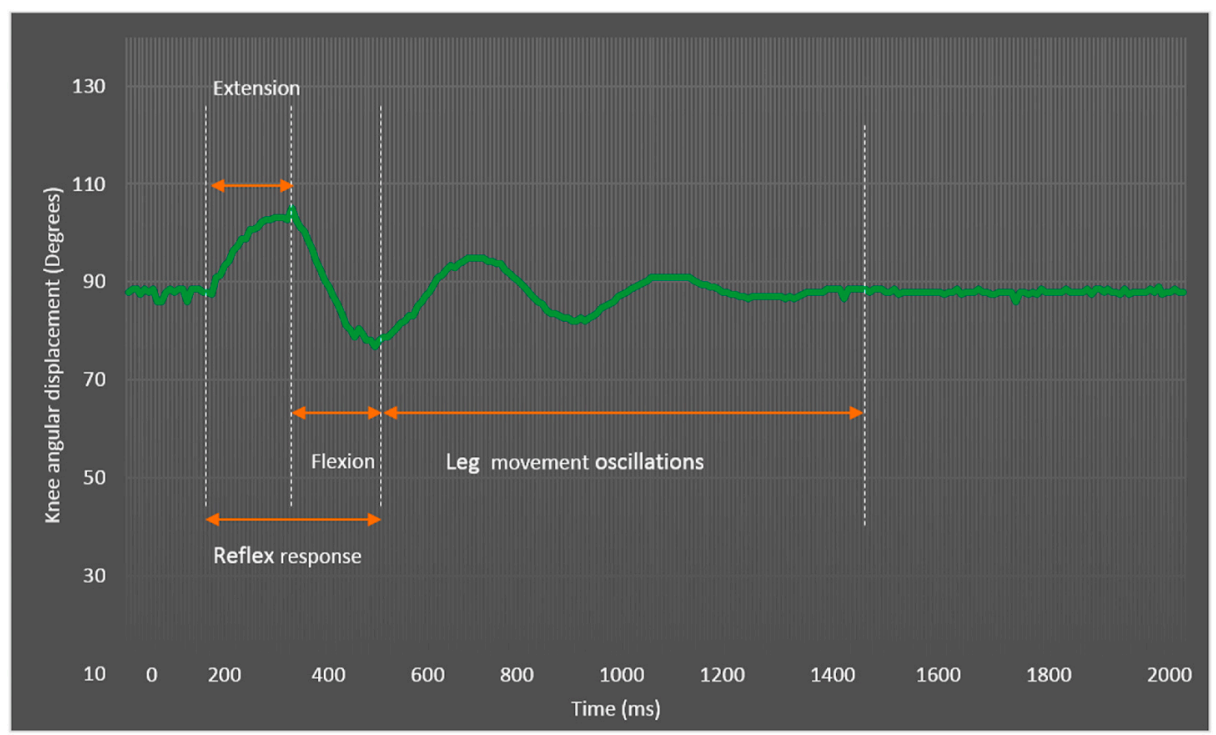

Fig. 3. Knee angular displacement during the PTR response

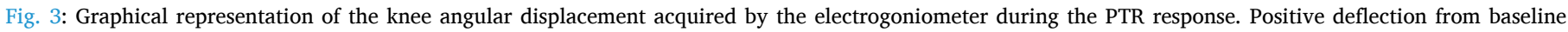
indicates extension and negative deflection is due to flexion at the knee. 
Quantified PTR test was characterized into dynamic variables (Table 1) resulted as a consequence of the reflex response.

\subsection{Statistical analysis}

The data sets were analyzed using SPSS 16 and the data sets were expressed as mean $\pm \mathrm{SD}$. The normality of data was tested using Kolmogorov- Smirnov test. As the data sets were skewed, non-parametric tests were applied to determine the associations between desired variables. The Spearman correlation was used to find out the relationship between PTR variables.

\section{Results}

The mean age of the participants was $32 \pm 5.5$ years. The mean height, weight and BMI of participants were $162.5 \pm 11.6 \mathrm{~cm}, 69.18 \pm$ $13.7 \mathrm{~kg}$ and $26.61 \pm 6.3$ respectively. The hip-length of each participant was $45.13 \pm 5.3 \mathrm{~cm}$ as summarized in Table 2 . According to the PTR test results using NINDS scale, none of the participants fell into grade $0+$ and grade $4+$. The phase - I result of PTR assessment showed high inter-rater variability and significant inter-knee PTR test variability (Table 3). This variability might be due to subjective visual assessment of the PTR response by clinicians. The objective assessment during phase - II using the PTR quantification system reveals that the tendon tapping force has shown statistically non-significant variation between the assessors, proving that constant tapping force was applied to achieve repeatability. The inter-knee PTR test variation was very minimal in the responses evaluated by the assessor -1 except two variables such as TPTR(ms) ( $p$ $<0.01 *)$ and EMG amp (mV) $(p-0.04 *)$ as shown in Table 4 . The results in Table -5 shows that the assessor -2 has got more variability in inter-knee PTR test assessment than assessor- 1 concerning the variables like $\mathrm{T}_{1}(\mathrm{~ms})$., $\operatorname{PEA}\left({ }^{\mathrm{O}}\right)$., $\operatorname{PEAD}\left({ }^{\mathrm{O}}\right)\left(p-0.04^{*}\right)$., $\mathrm{AV}(\mathrm{rad} / \mathrm{sec})\left(p<0.01^{*}\right)$ and

Table 1

Characterization of PTR response.

\begin{tabular}{|c|c|c|c|}
\hline $\begin{array}{l}\text { S. } \\
\text { No }\end{array}$ & Outcome variables & Units & Definition \\
\hline 1. & $\begin{array}{l}\text { Movement latency } \\
\text { (ML) }\end{array}$ & Milliseconds & $\begin{array}{l}\text { Duration from the point of tendon } \\
\text { tap to the onset of first movement } \\
\text { of leg into extension. }\end{array}$ \\
\hline 2. & $\begin{array}{l}\text { Total PTR response } \\
\text { time (TPTR) }\end{array}$ & Milliseconds & $\begin{array}{l}\text { Duration of total deep tendon } \\
\text { reflex response. }\end{array}$ \\
\hline 3. & Resting angle (RA) & Degrees & $\begin{array}{l}\text { The absolute angle at the Knee or } \\
\text { Ankle in resting position }\end{array}$ \\
\hline 4. & $\begin{array}{l}\text { Peak extension } \\
\text { angular displacement } \\
\text { (PEAD) }\end{array}$ & Radians & $\begin{array}{l}\text { Maximum angular change in the } \\
\text { response curve from the baseline } \\
\text { to the peak (Degrees of knee } \\
\text { extension) }\end{array}$ \\
\hline 5. & $\begin{array}{l}\text { Peak flexion angular } \\
\text { displacement (PFAD) }\end{array}$ & Radians & $\begin{array}{l}\text { Maximum angular change in the } \\
\text { response curve from the peak to } \\
\text { the baseline (Degrees of knee } \\
\text { flexion) }\end{array}$ \\
\hline 6. & Angular velocity (AV) & $\mathrm{rad} / \mathrm{sec}$ & $\begin{array}{l}\text { Rate of angular displacement } \\
\text { occurring due to biomechanical } \\
\text { movement during PTR response }\end{array}$ \\
\hline 7. & $\begin{array}{l}\text { Peak -to- Peak } \\
\text { amplitude }\end{array}$ & Millivolts & $\begin{array}{l}\text { Difference between highest } \\
\text { amplitude value and lowest } \\
\text { amplitude value }\end{array}$ \\
\hline 8. & $\begin{array}{l}\text { Time delay to first } \\
\text { peak }\left(\Delta \mathrm{t}_{1}\right)\end{array}$ & Milliseconds & $\begin{array}{l}\text { Time interval from the onset of } \\
\text { tendon strike to the first peak in } \\
\text { the response curve }\end{array}$ \\
\hline 9. & $\begin{array}{l}\text { Time delay to second } \\
\text { peak }\left(\Delta t_{2}\right)\end{array}$ & Milliseconds & $\begin{array}{l}\text { Time interval from the onset of } \\
\text { tendon strike to the second peak in } \\
\text { the response curve }\end{array}$ \\
\hline 10. & $\begin{array}{l}\text { Reflex mediated peak } \\
\text { EMG amplitude }\end{array}$ & Millivolts & $\begin{array}{l}\text { Reflex-mediated peak EMG signal } \\
\text { of the quadriceps muscles }\end{array}$ \\
\hline 11. & $\begin{array}{l}\text { Tendon tap force } \\
\text { (TTF) }\end{array}$ & Newtons & $\begin{array}{l}\text { Degree of impact force measured } \\
\text { during tendon striking }\end{array}$ \\
\hline
\end{tabular}

Table 1 Describing static and dynamic variables of PTR reaction response and their physiological role in interpretation.
Table 2

Demographics of participants.

\begin{tabular}{llll}
\hline Demographic variables & Mean \pm SD & CI of Mean & Range \\
\hline Age (Years) & $32.13 \pm 5.5$ & $30.3-33.8$ & $21-40$ \\
Gender (M/F) & $25 / 15$ & - & - \\
Height $(\mathrm{cm})$ & $162.5 \pm 11.6$ & $158.7-166.2$ & $140-181$ \\
Weight $(\mathrm{Kg})$ & $69.18 \pm 13.7$ & $64.7-73.5$ & $45-99$ \\
BMI & $26.61 \pm 6.3$ & $24.5-28.6$ & $14.2-42.1$ \\
Hip length $(\mathrm{cm})$ & $45.13 \pm 5.3$ & $43.4-46.8$ & $35-57$ \\
\hline
\end{tabular}

Table 2 Shows the demographic characteristics of the participants.

Table 3

Allocation of participants based on objective PTR response grading.

\begin{tabular}{|c|c|c|c|c|}
\hline \multirow[b]{2}{*}{ NINDS grading } & \multicolumn{2}{|l|}{ Clinician 1} & \multicolumn{2}{|l|}{ Clinician 2} \\
\hline & Right PTR & Left PTR & Right PTR & Left PTR \\
\hline Grade $0+$ & 0 & 0 & 0 & 0 \\
\hline Grade $1+$ & 7 & 2 & 9 & 3 \\
\hline Grade 2+ & 31 & 28 & 16 & 19 \\
\hline Grade 3+ & 2 & 10 & 15 & 18 \\
\hline Grade 4+ & 0 & 0 & 0 & 0 \\
\hline
\end{tabular}

Table 3: Distribution of participants based on PTR response grading assessed by ordinal NINDS reflex grading scale.

Table 4

Quantified PTR response characteristics by assessor - 1 .

\begin{tabular}{|c|c|c|c|}
\hline & $\begin{array}{l}\text { Right PTR }(\mathrm{n}=40) \\
\text { Mean } \pm \text { SD }\end{array}$ & $\begin{array}{l}\text { Left PTR }(\mathrm{n}=40) \\
\text { Mean } \pm \text { SD }\end{array}$ & $p$-Value \\
\hline TTF (Newtons) & $39 \pm 7.7$ & $38 \pm 6$ & 0.64 \\
\hline ML (ms) & $174.6 \pm 39.5$ & $172.8 \pm 39.7$ & 0.79 \\
\hline $\mathrm{RL}(\mathrm{ms})$ & $24.9 \pm 3.5$ & $26.3 \pm 5.1$ & 0.15 \\
\hline$\Delta \mathrm{t}_{1}(\mathrm{~ms})$ & $537 \pm 109.4$ & $546.8 \pm 94.6$ & 0.77 \\
\hline$\Delta \mathrm{t}_{2}(\mathrm{~ms})$ & $1410 \pm 262.7$ & $1407 \pm 216.2$ & 0.83 \\
\hline TTE (ms) & $370 \pm 117.2$ & $372 \pm 99.4$ & 0.98 \\
\hline $\mathrm{RA}\left({ }^{\mathrm{O}}\right)$ & $96.5 \pm 5.14$ & $98.2 \pm 6.2$ & 0.24 \\
\hline PEA $\left({ }^{\circ}\right)$ & $102.5 \pm 5.3$ & $104.1 \pm 6.5$ & 0.26 \\
\hline $\operatorname{PFA}\left({ }^{\circ}\right)$ & $93.8 \pm 6.4$ & $92.7 \pm 9$ & 0.57 \\
\hline $\operatorname{PEAD}\left({ }^{\mathrm{O}}\right)$ & $5.9 \pm 0.52$ & $5.8 \pm 3.5$ & 1.0 \\
\hline $\operatorname{PFAD}\left({ }^{\mathrm{O}}\right)$ & $8.73 \pm 4.6$ & $11.3 \pm 8.1$ & 0.09 \\
\hline $\mathrm{AV}$ (rad/sec) & $1.78 \pm 0.09$ & $1.8 \pm 0.1$ & 0.25 \\
\hline TPTR(ms) & $2293 \pm 467.8$ & $2665 \pm 743.6$ & $<0.01 *$ \\
\hline $\begin{array}{l}\text { EMG peak amp } \\
\qquad(\mathrm{mV})\end{array}$ & $0.73 \pm 0.18$ & $0.65 \pm 0.11$ & $<0.04 *$ \\
\hline
\end{tabular}

Table 4: Characteristics of bilateral PTR response of participants evaluated by assessor 1 .

Table 5

Quantified PTR response characteristics by assessor - 2.

\begin{tabular}{|c|c|c|c|}
\hline & $\begin{array}{l}\text { Right PTR }(\mathrm{n}=40) \\
\text { Mean } \pm \text { SD }\end{array}$ & $\begin{array}{l}\text { Left PTR }(n=40) \\
\text { Mean } \pm \text { SD }\end{array}$ & $\begin{array}{l}p- \\
\text { Value }\end{array}$ \\
\hline TTF (Newton) & $29.5 \pm 9.6$ & $30.7 \pm 8.8$ & 0.57 \\
\hline ML (ms) & $168.7 \pm 47.1$ & $186.5 \pm 64$ & 0.16 \\
\hline $\mathrm{RL}(\mathrm{ms})$ & $23.1 \pm 5.6$ & $26.2 \pm 6.1$ & 0.02 \\
\hline$\Delta \mathrm{t}_{1}(\mathrm{~ms})$ & $466.8 \pm 65.3$ & $501.7 \pm 86.8$ & $0.04 *$ \\
\hline$\Delta \mathrm{t}_{2}(\mathrm{~ms})$ & $1377 \pm 285.6$ & $1358 \pm 274$ & 0.76 \\
\hline TTE (ms) & $316.8 \pm 67.6$ & $315.5 \pm 79.9$ & 0.93 \\
\hline $\mathrm{RA}\left({ }^{\mathrm{O}}\right)$ & $97 \pm 4.8$ & $97.8 \pm 6$ & 0.49 \\
\hline PEA $\left({ }^{\circ}\right)$ & $103.8 \pm 4.2$ & $106.4 \pm 6.6$ & $0.04 *$ \\
\hline $\operatorname{PFA}\left({ }^{\circ}\right)$ & $93 \pm 6$ & $93.8 \pm 9.1$ & 0.66 \\
\hline $\operatorname{PEAD}\left({ }^{\mathrm{O}}\right)$ & $6.8 \pm 3$ & $8.4 \pm 3.1$ & 0.01 * \\
\hline PFAD $\left({ }^{\circ}\right)$ & $10.7 \pm 6.3$ & $12.5 \pm 9.6$ & 0.33 \\
\hline $\mathrm{AV}(\mathrm{rad} / \mathrm{sec})$ & $1.8 \pm 0.07$ & $1.85 \pm 0.11$ & $0.05 *$ \\
\hline TPTR(ms) & $2404 \pm 468.1$ & $2717 \pm 742$ & $0.02 *$ \\
\hline $\begin{array}{l}\text { EMG peak amp } \\
\qquad(\mathrm{mV})\end{array}$ & $0.70 \pm 0.19$ & $0.64 \pm 0.13$ & 0.11 \\
\hline
\end{tabular}

Table 5: Characteristics of bilateral PTR response of participants assessed by assessor 2 . 
Table 6

Inter-assessor variability of quantified PTR response.

\begin{tabular}{|c|c|c|c|c|c|c|}
\hline & Right PTR Tester 1 & Right PTR Tester 2 & $p$ - Value & Left PTR Tester1 & Left PTR Tester 2 & $p$ - Value \\
\hline TTF (Newton) & $39 \pm 7.7$ & $29.5 \pm 9.6$ & $<0.01 *$ & $38 \pm 6$ & $30.7 \pm 8.8$ & $<0.01 *$ \\
\hline ML (ms) & $174.6 \pm 39.5$ & $168.7 \pm 47.1$ & 0.51 & $172.8 \pm 39.7$ & $186.5 \pm 64$ & 0.25 \\
\hline $\mathrm{RL}(\mathrm{ms})$ & $24.9 \pm 3.5$ & $23.1 \pm 5.6$ & 0.10 & $26.3 \pm 5.1$ & $26.2 \pm 6.1$ & 0.96 \\
\hline$\Delta \mathrm{t}_{1}(\mathrm{~ms})$ & $537 \pm 109.4$ & $466.8 \pm 65.3$ & $<0.01$ * & $546.8 \pm 94.6$ & $501.7 \pm 86.8$ & $0.02 *$ \\
\hline$\Delta \mathrm{t}_{2}(\mathrm{~ms})$ & $1410 \pm 262.7$ & $1377 \pm 285.6$ & 0.49 & $1407 \pm 216.2$ & $1358 \pm 274$ & 0.37 \\
\hline TTE (ms) & $370 \pm 117.2$ & $316.8 \pm 67.6$ & 0.01 & $372 \pm 99.4$ & $315.5 \pm 79.9$ & $<0.05^{*}$ \\
\hline $\mathrm{RA}\left({ }^{\mathrm{O}}\right)$ & $96.5 \pm 5.14$ & $97 \pm 4.8$ & 0.79 & $98.2 \pm 6.2$ & $97.8 \pm 6$ & 0.78 \\
\hline $\operatorname{PEA}\left({ }^{\circ}\right)$ & $102.5 \pm 5.3$ & $103.8 \pm 4.2$ & 0.24 & $104.1 \pm 6.5$ & $106.4 \pm 6.6$ & 0.13 \\
\hline PFA $\left({ }^{\circ}\right)$ & $93.8 \pm 6.4$ & $93 \pm 6$ & 0.62 & $92.7 \pm 9$ & $93.8 \pm 9.1$ & 0.60 \\
\hline $\operatorname{PEAD}\left({ }^{\mathrm{O}}\right)$ & $5.9 \pm 0.52$ & $6.8 \pm 3$ & 0.19 & $5.8 \pm 3.5$ & $8.4 \pm 3.1$ & $<0.01 *$ \\
\hline PFAD $\left({ }^{\circ}\right)$ & $8.73 \pm 4.6$ & $10.7 \pm 6.3$ & 0.12 & $11.3 \pm 8.1$ & $12.5 \pm 9.6$ & 0.54 \\
\hline $\mathrm{AV}(\mathrm{rad} / \mathrm{sec})$ & $1.78 \pm 0.09$ & $1.8 \pm 0.07$ & 0.24 & $1.8 \pm 0.1$ & $1.85 \pm 0.11$ & 0.13 \\
\hline TPTR(ms) & $2293 \pm 467.8$ & $2404 \pm 468.1$ & 0.37 & $2665 \pm 743.6$ & $2717 \pm 742$ & 0.75 \\
\hline EMG peak amp (mV) & $0.73 \pm 0.18$ & $0.70 \pm 0.19$ & 0.53 & $0.65 \pm 0.11$ & $0.64 \pm 0.13$ & 0.58 \\
\hline
\end{tabular}

* $p$ value is significant.

Table 6: Shows the inter-observer difference of quantitative reflex characteristics evaluated by the trained assessors using the PTR quantification system.

TPTR(ms) $(p-0.02 *)$. However, analysis of quantified PTR variables proved to be better than semi-quantitative assessment and indicate that the proposed system and methodology are reproducible for objective PTR assessment. Table -6 , reveals the inter-observer variability for quantified PTR between two assessors. The rate of error would be less in digitally quantified variables compared with the results of ordinal reflex rating scale. All PTR variables have shown an insignificant difference between assessors except $\mathrm{T}_{1}(\mathrm{~ms})$, TTE $(\mathrm{ms})$ and PEAD $\left({ }^{\mathrm{O}}\right)\left(p<0.05^{*}\right)$. This could be due to the unavoidable control on minute changes in TTF, and the variability is in milliseconds which could not affect the overall outcome of PTR evaluation.

\section{Discussion}

This study describes the significance of quantitative measures of PTR test utilizing an indigenously developed digital technique. The accuracy and reproducibility of the device are evaluated by a strategic protocol that reveals the extent of variability. In this study, quantified PTR response was characterized into multiple variables, which would be helpful for clinicians in decision making during the assessment of patient's neurological condition. Assessment of PTR response can be used as a screening tool to identify potential neurological deficits and evaluate the severity of neurological disorders in clinical practice. ${ }^{20}$ Observation of alterations in the tendon reflex response has a predictive value and can greatly aid the diagnosis of neurological disorders. ${ }^{21}$ Various neurological and endocrine disorders influencing the magnitude of tendon reflex responses. ${ }^{22}$ Though the evaluation of PTR has clinical importance, its utility is uncertain in practice due to its subjective assessment and high inter-observer variability. ${ }^{12}$ In this study, quantification of PTR test was performed using wearable biosensors. Many objective methods reported for the quantitative evaluation of PTR response in the last few decades due to advancement in technology and the availability of activity monitoring body-worn biosensors. Abazova et al. (2005) developed a direct method for measuring knee jerk's reflex time in thyroid patients using reflexometry technique consisting of a solenoid sensor connecting to an electrocardiograph. ${ }^{23}$ However, this technique has some disadvantages such as the complexity of the device and high measuring error. Reflex latency and amplitude of PTR response described in a study conducted by Frinjs et al. (1997) using electromyography (EMG) in healthy volunteers to establish normative data of patellar and ankle reflexes. ${ }^{24}$ Sharma et al. (2007) conducted a study using surface EMG to establish the diagnostic value of reflex latency measurement. ${ }^{25}$ However, recent studies concluded that the use of traditional EMG or surface EMG has some limitations such as power line interference, skin preparation and restricted use outside the laboratory environment. ${ }^{26}$ The proposed system used in this study does not require a large laboratory setup or skin preparation. In a more recent study done by Tsuji et al. (2021), researchers used Mechanomyography (MMG) and Electromyography (EMG) devices to objectively characterize PTR response which could be helpful in clinical practice to diagnose neurological disorders. ${ }^{27}$ In a study by Kim YW (2015), a novel DTR apparatus was designed to assess its clinical utility using goniometric measurements. ${ }^{28}$ This study employed an electrogoniometer to measure the knee angular displacement and angular velocity. Salazar et al. (2019) utilized a device for measurement of angular velocity of the leg during reflex response using machine learning classifiers. Mamizuka et al. (2007) used a triaxial accelerometer to demonstrate the difference in PTR response between myelopathy patients and healthy volunteers. The proposed PTR quantification unit also comprises an accelerosensor for a better outcome, as these sensors can provide reliable data of movement during PTR response. Previous research studies conducted by Sullivan et al. (2015) and Tham L.K et al. (2013) showed that PTR response quantitative measures could be effectively obtained using the motion analysis technique. However, the motion analysis technique is considered as an expensive tool and may not be feasible for field studies. Previous research concluded that factors like age, gender, and height believed to influence the reflex response. ${ }^{29},{ }^{30}$ However, in this study, these factors did not significantly influence the characteristics of PTR response. According to the studies done by Marshal et al. (2002), the most significant factor which could influence the tendon reflex response is the tendon tapping force, and the authors of the study suggested a tapping force of $21-50 \mathrm{~N}$ is appropriate to elicit normorelexic response. In the current study, the tendon tapping force applied by trained assessors using customized hammer yielded an almost similar range of tapping force (Assessor 1; 28-62 N and Assessor 2; 13-50 N). In another study done by Serkan Uslu et al. (2016) has concluded that the relationship between tendon tapping force and ordinal DTR grading was difficult to establish. ${ }^{31}$ Our study also could not able to find the association between tendon tapping force and desired PTR variables. The mass of the hammer $(200 \mathrm{~g})$ was constant throughout the study which may not be a confounding factor. Hence, it is consequently assumed that the tapping force was not significantly variable between assessors during assessment process. Inter - knee variability was observed between the assessors in respect to few parameters which could be resulted due to biological variation. Our study reveals that the PTR responses were accurately characterized by quantitative variables such as ML, TPTR, PEAD, PFAD and AV. Analysis of these quantitative variables may be beneficial for clinical assessment because they represent the integrity of reflex arc and movement characteristics of knee during tendon reflex response.

\subsection{Strengths \& limitations}

The current study describes the design and development of an in- 
house built digital apparatus to quantify the PTR responses. The device is entirely non-invasive, and there are no anticipated sudden adverse effects. It is more convenient, user friendly and does not cause any discomfort to the subject. Its portability makes it simple to collect data in community studies, and the time required to record and report is very minimal. In this study, the robustness of the device was established. However, there are certain limitations of the current study. The device usage requires minimal computer knowledge. The influence of age, gender and other elements on reflex response did not establish in this study.

\section{Conclusion}

The proposed PTR quantification system is a valid and reliable objective method to assess patellar tendon reflex response. Implementation of this approach to evaluate the other DTRs can enhance the accuracy and reliability of determining reflex responses in clinical settings. The proposed clinical method for assessing tendon reflexes (Muscle stretch reflexes) warrants study in patients with altered tendon reflex responses. Nonetheless, by integrating future methodological enhancements such as wireless sensors and technology for accessible data processing, the proposed system might lead to full automatization of DTR assessment.

\section{Funding}

This research did not receive any specific grant from funding agencies in the public, commercial, or not-for-profit sectors.

\section{CRediT authorship contribution statement}

S.K. Kareem: Conceptualization, Data collection, Data processing, Writing - original draft. Dilara K: Conceptualization, Supervision, Writing - original draft. K.N. Maruthy: Conceptualization, Methodology, creation of models. Priscilla Johnson: Supervision, Ideas. A.V. Siva Kumar: Resources, Formal analysis, Writing - review \&; editing.

\section{Acknowledgement}

The authors would like to express their sincere gratitude to the University authorities of Sri Ramachandra Institute of higher education \& Research, Chennai and Management of Narayana Medical College \& Hospital, Nellore for providing basic amenities and support during the study period.

\section{References}

1 Boes CJ. The history of examination of reflexes. J Neurol. 2014 Dec;261(12): 2264-2274.

2 Dick JP. The deep tendon and the abdominal reflexes. J Neurol Neurosurg Psychiatr. 2003 Feb 1;74(2):150-153.

3 O'Sullivan R, Kiernan D, Walsh M, O'Brien T, Elhassan Y. Characterisation of the patellar tendon reflex in cerebral palsy children using motion analysis. Ir J Med Sci. 2016 Nov;185(4):813-817.

4 Bhattacharyya KB. Deep tendon reflex: the background story of a simple technique. Neurol India. 2017 Mar 1;65(2):245.

5 Marshall GL, Little JW. Deep tendon reflexes: a study of quantitative methods. The journal of spinal cord medicine. 2002 Jul 1;25(2):94-99.

6 Salazar-Muñoz Y, López-Pérez GA, García-Caballero BE, Muñoz-Rios R, RuanoCalderon LA, Trujillo L. Classification and assessment of the patelar reflex response through biomechanical measures. Journal of healthcare engineering. 2019 Jul 9:2019.
7 Dafkin C, Green A, Kerr S, Veliotes D, Mckinon W. The accuracy of subjective clinical assessments of the patellar reflex. Muscle Nerve. 2013 Jan;47(1):81-88.

8 Lemoyne R, Dabiri F, Jafari R. Quantified deep tendon reflex device, second generation. J Mech Med Biol. 2008 Mar;8(1):75-85.

9 Stam J, Van Crevel H. Measurement of tendon reflexes by surface electromyography in normal subjects. J Neurol. 1989 May;236(4):231-237.

10 LeMoyne R, Kerr WT, Zanjani K, Mastroianni T. Implementation of an iPod wireless accelerometer application using machine learning to classify disparity of hemiplegic and healthy patellar tendon reflex pair. Journal of medical imaging and health informatics. 2014 Mar 1;4(1):21-28.

11 Steineman BD, Karra P, Park K. Assessment of patellar tendon reflex responses using second-order system characteristics. Appl Bionics Biomechanics. 2016 Feb 14:2016.

12 Manschot S, Van Passel L, Buskens E, Algra A, Van Gijn J. Mayo and NINDS scales for assessment of tendon reflexes: between observer agreement and implications for communication. J Neurol Neurosurg Psychiatr. 1998 Feb 1;64(2):2535.

13 Zhang LQ, Huang H, Sliwa JA, Rymer WZ. System identification of tendon reflex dynamics. IEEE Trans Rehabil Eng. 1999 Jun;7(2):193-203.

14 Shaik K, Kamaldeen D, Maruthy KN, Johnson P, Kumar AS. A newer approach for quantitative assessment of patellar tendon reflex response using biomechanical data of foot movement by a digital method. Sports Orthopaedics and Traumatology. 2020 Sep 1;36(3):271-277. https://doi.org/10.1016/j.orthtr.2020.04.010.

15 Zhang L, Huang H, Nishida T, Zeng K, Ballantyne B, Rymer WZ. Quantification of tendon reflex in normal and spastic limbs. InProceedings of the 19th Annual International Conference of the IEEE Engineering in Medicine and Biology Society.'Magnificent Milestones and Emerging Opportunities in Medical Engineering'(Cat. No. 97CH36136) 1997 Dec 1 (Vol. 5, pp. 1958-1961). IEEE

16 Zimmerman B, Hubbard JB. Deep tendon reflexes. 2020 Jul 31. In: StatPearls [Internet]. Treasure Island (FL: StatPearls Publishing; 2021 Jan. PMID: 30285397.

17 Litvan I, Mangone CA, Werden W, et al. Reliability of the NINDS myotatic reflex scale. Neurology. 1996 Oct 1;47(4):969-972.

18 Tham LK, Abu Osman NA, Wan Abas WA, Lim KS. The validity and reliability of motion analysis in patellar tendon reflex assessment. PLoS One. 2013 Feb 7;8(2), e55702.

19 Hermens HJ, Freriks B, Disselhorst-Klug C, Rau G. Development of recommendations for SEMG sensors and sensor placement procedures. J Electromyogr Kinesiol. 2000 Oct 1;10(5):361-374.

20 Mamizuka N, Sakane M, Kaneoka K, Hori N, Ochiai N. Kinematic quantitation of the patellar tendon reflex using a tri-axial accelerometer. J Biomech. 2007 Jan 1;40(9): 2107-2111.

21 Ohtaki Y, Mamizuka N, Fard M, Harada Y, Ochiai N. BIO-06 A handy instrument for quantitative evaluation $\mathrm{OF}$ kinematic patellar tendon reflex responses (Bio-medical equipments II, technical program of oral presentations).InProceedings of JSME-IIP/ ASME-ISPS Joint Conference on Micromechatronics for Information and Precision Equipment: IIP/ISPS Joint MIPE 2009 2009 Jun 17 (pp. 225-226). The Japan Society of Mechanical Engineers.

22 Chardon MK, Rymer WZ, Suresh NL. Quantifying the deep tendon reflex using varying tendon indentation depths: applications to spasticity. IEEE Trans Neural Syst Rehabil Eng. 2014 Jan 13;22(2):280-289.

23 Abazova ZK, Baisiev AK, Kumykov VK, Efendieva MK. Direct method for measuring reflex time of knee jerk in patients with thyroid pathologies. Biomedical Engineering. 2006 May 1;40(3):117.

24 Frijns CJ, Laman DM, Van Duijn MA, Van Duijn H. Normal values of patellar and ankle tendon reflex latencies. Clin Neurol Neurosurg. 1997 Feb 1;99(1):31-36.

25 Sharma KR, Saadia D, Facca AG, Resnick S, Ayyar DR. Clinical and electromyographic deep tendon reflexes in polyneuropathy: diagnostic value and prevalence. Acta Neurol Scand. 2009 Apr;119(4):224-232.

26 Woodward RB, Stokes MJ, Shefelbine SJ, Vaidyanathan R. Segmenting mechanomyography measures of muscle activity phases using inertial data. Sci Rep. 2019 Apr 3;9(1):1.

27 Tsuji H, Misawa H, Takigawa T, et al. Quantification of patellar tendon reflex using portable mechanomyography and electromyography devices. Sci Rep. 2021 Jan 27; 11(1):1-5.

28 Kim YW. Clinical availability of the deep tendon reflex test using a novel apparatus in healthy subjects. J Phys Ther Sci. 2015;27(2):317-320.

29 Chandrasekhar A, Abu Osman NA, Tham LK, Lim KS, Wan Abas WA. Influence of age on patellar tendon reflex response. PLoS One. 2013 Nov 18;8(11), e80799.

30 Burke JR, Koceja DM, Kamen G. The relationship between body height extremes and the conditioned patellar tendon reflex response. Int J Neurosci. 1993 Jan 1;72(1-2): 45-58.

31 Uslu S, Nüzket T, Uysal H. Modified motor unit number index (MUNIX) algorithm for assessing excitability of alpha motor neuron in spasticity. Clinical neurophysiology practice. 2018 Jan 1;3:127-133. 